\title{
LA RED ESPAÑOLA DE ALTA VELOCIDAD FERROVIARIA: ANÁLISIS MEDIANTE UN MODELO GRAVITACIONAL ${ }^{1}$.
}

\section{SPANISH NETWORK HIGH SPEED RAIL: ANALYSIS USING A GRAVITY MODEL.}

\author{
Sergio Martín Cabo \\ Universidad Complutense de Madrid \\ sergiomc84@gmail.com \\ Recibido: marzo, 2012. \\ Versión final aceptada: abril, 2013.
}

PALABRAS CLAVE: red de alta velocidad, modelo gravitatorio, masa demográfica, distancia, pasajeros.

KEYWORDS: high speed network, gravitational model, demographic mass, distance, passengers.

\section{RESUMEN}

Desde los albores del siglo XXI, el tren de alta velocidad se encuentra en pleno proceso de expansión a nivel nacional, incrementando muy significativamente los kilómetros de red así como el número de ciudades conectadas a la misma. Mediante la aplicación de un modelo gravitacional, se pretenden exponer en la presente comunicación cómo incide esa expansión a nivel de volumen de tráfico de pasajeros, teniendo en cuenta masas demográficas y distancias espaciales, detectando corredores con una importante demanda potencial mientras que otros cuentan con ciertas disfuncionalidades, que inciden negativamente en el flujo de usuarios.

\section{ABSTRACT}

Since the dawn of the century, the high-speed train is in the process of expanding nationwide, increasing very significantly the miles of network and the number of cities connected to it. By applying a gravity model, is intended to present in this communication how expansion affects the level of passenger traffic volume, mass considering demographic and spatial distances, detecting corridors with significant potential demand while others have certain dysfunctions, that adversely affect the flow of users.

\footnotetext{
1 El presente artículo procede de la comunicación científica presentada en las Jornadas de Estudios Regionales: Redes y sus territorios: Incidencia en el desarrollo regional. AGE. Sevilla, Junio de 2012.
}

ISSN: 0212-8594 ISSN-e: 2340-2776. № DOI: http://dx.doi.org/10.12795/rea.2013.i30.04 


\section{INTRODUCCIÓN.}

La red ferroviaria de alta velocidad española se encuentra en pleno proceso de crecimiento. Tras la inauguración de la primera línea de alta velocidad (en adelante LAV) en abril de 1992, para conectar Madrid con Sevilla, siguió una década de estancamiento. Dicho decenio finalizó con la inauguración en 2003 del trazado MadridLleida, fase anterior a la conexión de la capital con Barcelona y (más a largo plazo) con la frontera francesa. Tras esta inauguración prosiguieron otras: LAV Zaragoza-Huesca (2003), LAV Madrid-Toledo (2005), LAV Córdoba-Málaga (2007), LAV Madrid-Valladolid (2007), LAV Lleida-Barcelona (2008),LAV Madrid-Valencia (2010) y finalmente el corredor gallego A Coruña-Ourense (2011), que conectará en un futuro con Valladolid, configurándose por tanto como el eje ferroviario noroeste de alta velocidad.

Estas sucesivas ampliaciones, junto a los proyectos ya en curso, posibilitan que España sea uno de los países con una red más amplia a nivel mundial, lo cual genera múltiples implicaciones de toda índole que pueden abordarse desde diferentes perspectivas temáticas: económica, ambiental, política, social, urbanística; y, en definitiva, territorial.

Del mismo modo, esta multidisciplinariedad científica a la hora de realizar análisis referentes a dicha red se refleja en la multiplicidad de metodologías empleadas. En el presente trabajo se va a analizar la red española de alta velocidad ferroviaria mediante la utilización de los denominados modelos gravitatorios, concretamente el modelo gravitatorio de Archer.

Según Denise Pumain, profesora de la Universidad de La Sorbona y experta en la aplicación de modelos matemáticos para investigaciones de carácter urbano, un modelo gravitatorio es aquel destinado a formalizar, estudiar y prever la geografía de los flujos o de las interacciones. Deriva de la ley de gravitación universal de Newton: dos cuerpos se atraen en razón directa de su masa y en razón inversa de la distancia que los separa. Asimismo, en un espacio de circulación relativamente homogéneo, cuanto mayores sean los intercambios entre dos regiones o ciudades, mayor será su peso; de igual forma, si éstas están más alejadas, los intercambios serán más débiles. (Pumain, D., 2004).

Existen numerosos autores que han formulado modelos de gravitación (Crampon, Ellis y Van Doren, Gordon y Edwards, Armstrong, etc.). Sin embargo, B. Archer es quizá el autor más destacado en cuanto a su aplicación a la dimensión territorial. Dicho autor estableció un modelo dirigido a medir el efecto económico del turismo y sus impactos directo, indirecto e inducido en la economía donde se producen, así como la aportación realizada por el turismo a los restantes sectores económicos. Además de ello, fue pionero en materia de multiplicadores keynesianos aplicados al gasto turístico 
también desde un punto de vista territorial (Muñoz, A. et al., 1994; Calderón, F.J.,2007).

Tomando como base parte de la formulación de Archer se ha creado un modelo destinado al análisis de la red de alta velocidad existente en servicio en España.

\section{OBJETO Y METODOLOGÍA.}

El objetivo de este trabajo es el de determinar el posible tráfico de viajeros que utiliza el tren de alta velocidad, mediante la aplicación de un modelo gravitacional y desglosando los resultados por tramos de línea.

Para la realización del presente análisis se han tenido en cuenta las estaciones de alta velocidad existentes a fecha de abril de 2012, las cuales ascienden a un total de 26, siendo las siguientes: Madrid, Toledo, Ciudad Real, Puertollano (Ciudad Real), Córdoba, Sevilla, Puente Genil (Córdoba), Antequera (Málaga), Málaga, Guadalajara, Calatayud (Zaragoza), Zaragoza, Lleida, Tarragona, Barcelona, Tardienta (Huesca), Huesca, Segovia, Valladolid, Cuenca, Albacete, Requena-Utiel (Valencia), Valencia, A Coruña, Santiago de Compostela (A Coruña) y Ourense.

Todas estas estaciones cuentan con servicios de alta velocidad de Larga Distancia (bajo las marcas comerciales AVE y Alvia) o bien de Media Distancia (corredor gallego, Toledo, Tardienta y Huesca), en cuyo caso la marca comercial que opera es Avant. En relación a los trazados, se han considerado aquellos que ofertan servicios de alta velocidad ${ }^{2}$.

Los indicadores que se han tenido en cuenta a la hora de la realización del análisis se resumen en dos tipos principales. En primer lugar, variables demográficas, considerando la población de cada unidad provincial y de cada uno de los municipios que albergan una estación ferroviaria de alta velocidad. Dichas cifras han sido extraídas del Instituto Nacional de Estadística y hacen referencia a la población existente a 1 de enero de 2011. En segundo lugar, se han considerado las distancias kilométricas existentes entre todas las estaciones mediante trayectos que discurren por los trazados de alta velocidad.

A la hora de analizar la relación de enlaces existentes, únicamente se han considerado aquellos cuya conexión es directa, es decir, no existiendo la necesidad de hacer ningún trasbordo para el desplazamiento entre una estación $i$ y una estación $j$.

\footnotetext{
${ }^{2}$ Existen servicios de alta velocidad realizados por marcas comerciales como Euromed y Alaris, las cuales no se han considerado puesto que dichos servicios no se realizan en corredores de alta velocidad (por ejemplo Barcelona-Alicante, Albacete-Valencia (por Xátiva), etc.
}

ISSN: 0212-8594 ISSN-e: 2340-2776. № DOI: http://dx.doi.org/10.12795/rea.2013.i30.04 REA 30 (2013): 78-101

http://www.publius.us.es/estudios_andaluces 
Por otra parte, la formulación que se ha tenido en cuenta para la aplicación del modelo es la siguiente:

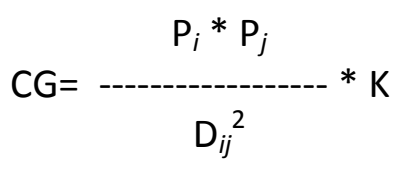

Siendo:

CG = Coeficiente Gravitacional.

$\mathrm{P}=$ Masa demográfica (Millones de habitantes)

ij $=$ Nodos a analizar en cada caso

$\mathrm{D}=$ Distancia (Kilómetros)

$\mathrm{K}=$ Constante, con valor de 0,66

A partir de esta simple formulación se plantean diferentes dificultades metodológicas, que implican una mayor complejidad a la hora de aplicar de forma más lógica y real el modelo.

Una primera dificultad hace referencia a la masa demográfica que debe considerarse en cada uno de los tramos analizados, derivado a su vez de las diferencias existentes entre las estaciones de alta velocidad y la cuantía poblacional a la que ofrece servicio cada una de éstas. Concretamente, el emplazamiento de cada una de las estaciones es vital para poder establecer una modelización sobre qué tráfico de viajeros puede tener.

Por ejemplo, no ofrecen la misma oferta "potencial" de servicio Barcelona-Sants que Guadalajara-Yebes. Pero tienen un rasgo identificatorio común: ambas son la única estación de alta velocidad de las respectivas provincias a las que pertenecen.

Otro ejemplo diferente es el caso de Ciudad Real y Puertollano. Las diferencias en relación a sus respectivas masas demográficas son pequeñas. Además de ello, ambas estaciones se encuentran a escasa distancia y en la misma provincia. Sin hacerlo mucho más complejo, dado que los flujos de viajeros no tienen porqué regirse exactamente por criterios político-administrativos, resulta que dicha provincia cuenta con dos estaciones, con lo cual deben repartirse la demanda de tráfico.

Como es natural, una estación de alta velocidad debe captar no sólo viajeros que habitan en el propio núcleo en el que se emplaza la estación, sino también de otros núcleos. En este sentido, la determinación del área de captación de viajeros de cada estación es una tarea extraordinariamente compleja, debido a la pluralidad de factores que convergen y a la escasez de datos disponibles. 
Tras este cálculo, se han pasado los valores de CG a valores de pasajeros. La idea es establecer unas primeras pautas para determinar, a partir de masa demográfica, distancia y coeficiente gravitatorio, qué masa potencial máxima anual va a tener cada enlace, dando por supuesto que el tráfico real será sensiblemente inferior. Es de carácter anual porque el modelo de Archer se aplica al año y, de forma excepcional, mensualmente, para analizar la estacionalidad. La expresión utilizada ha sido la siguiente:

$\operatorname{PAX}(\mathrm{M})=\left(\mathrm{P}_{i}+\mathrm{P}_{j}\right) * \mathrm{CG}_{i j} * \mathrm{~K}$

Siendo:

PAX (M) =Pasajeros máximos potenciales anuales (millones).

Se ha incluido una constante " $\mathrm{K}$ " al final de la expresión para mitigar las distorsiones en los resultados.

En el siguiente apartado se exponen los resultados de la aplicación a la red del modelo gravitatorio de Archer, la tipificación de estaciones realizada en función de éstos y la operativa seguida en cada una de las categorías.

\section{LA RED DE ALTA VELOCIDAD FERROVIARIA ESPAÑOLA.}

Como ya se ha expuesto anteriormente, el primer corredor de alta velocidad español empezó a gestarse a mediados de los años 80 con el planteamiento del NAFA (Nuevo Acceso Ferroviario a Andalucía) cuyo trazado discurriría evitando el paso de Despeñaperros (Jaén), concretamente $90 \mathrm{Km}$. al oeste. Formaría parte de lo que años más tarde, en 1992, sería el primer corredor de alta velocidad ferroviaria de España, conectando las ciudades de Madrid y Sevilla.

Con el cambio de siglo, la red comenzó a extenderse y no ha dejado de hacerlo hasta la actualidad, encontrándonos con los trazados representados en la figura 1. En este mapa también aparecen representadas en forma de coropletas la población de las provincias españolas con fecha de enero de 2011.

Puede establecerse una relación entre el sistema de ciudades español ( $y$, en consecuencia, la distribución de sus masas demográficas) con el carácter radial de la red de alta velocidad ferroviaria. Es sabido el desequilibrio demográfico existente en la península entre el centro y la periferia, con Barcelona, Valencia, Málaga, Alicante, Murcia, etc. erigiéndose como grandes ciudades mientras que el centro permanecía con unas densidades mucho más bajas, a excepción de Madrid, actuando como gran metrópoli central rodeada de un gran vacío poblacional. 
Pues bien, la capital fortalece su posición ejerciendo de principal nodo de comunicaciones en alta velocidad ferroviaria en una red marcada por su fuerte carácter radial. De igual forma, actúa tanto de cabecera como fin de línea de los principales corredores, excepto el corredor gallego, el cual dispondrá de una futura conexión a Valladolid y, por tanto, también a Madrid.

A excepción de la LAV de Galicia, el resto de corredores tienen conexión con Madrid, y la mayor parte de ellos cuentan con nodos de cabecera o final de líneas con unas masas demográficas muy superiores a las de las estaciones intermedias del interior, a

Figura 1. Red de alta velocidad (abril 2012) y distribución demográfica provincial.

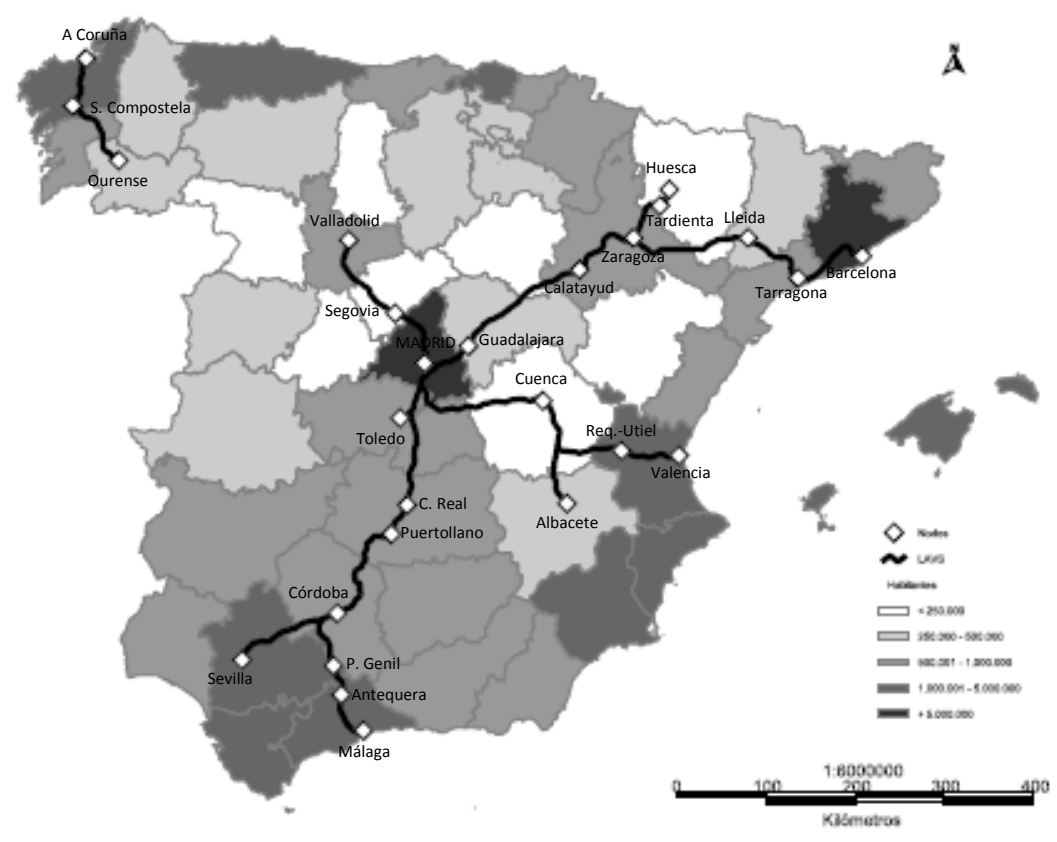

Fuente: Elaboración propia.

semejanza del sistema demográfico nacional y sus desequilibrios centro-periferia. Véase el caso de la LAV Madrid-Barcelona, cuyos nodos extremos son los más potentes de la red española en cuanto a población, mientras que nodos intermedios (especialmente entre Madrid y Zaragoza) se encuentran con unos valores demográficos absolutos mucho menores (Guadalajara y Calatayud). O bien la LAV Madrid-Sevilla, con nodos intermedios con menor entidad desde un punto de vista demográfico como son Ciudad Real y Puertollano; así como la LAV Madrid-Valencia, con las estaciones de Cuenca y Requena-Utiel.

Una excepción a lo anterior es la línea que conecta Madrid con Albacete, con un final de línea no periférico y no demasiado poblado. No obstante, Albacete es un nodo final

ISSN: 0212-8594 ISSN-e: 2340-2776. № DOI: http://dx.doi.org/10.12795/rea.2013.i30.04 REA 30 (2013): 78-101 
de línea provisional, ya que en la actualidad se están ejecutando las obras para la prolongación de dicho corredor hacia Alicante y Murcia, ciudades con masas demográficas notablemente superiores a la urbe manchega.

En el cuadro 1 se detallan los nodos objeto de estudio, las masas demográficas correspondientes a sus términos municipales así como a sus respectivas provincias. En la última columna se incluye la cuantía porcentual que representa la masa demográfica de cada nodo con respecto a la totalidad de su provincia correspondiente.

Cuadro 1. Distribución demográfica de las poblaciones objeto de estudio (2011).

\begin{tabular}{|c|c|c|c|}
\hline & MASA DEMOGRÁFIC & Millones de hab.) & \\
\hline NODO & PROVINCIAL & T. MUNICIPAL & \% Pob. T. M./Pob. PROV \\
\hline Madrid & 6,489680 & 3,265038 & 50,311 \\
\hline Toledo & 0,707242 & 0,083108 & 11,751 \\
\hline Ciudad Real & 0,530175 & 0,074798 & 14,108 \\
\hline Puertollano & 0,530175 & 0,052200 & 9,846 \\
\hline Córdoba & 0,805857 & 0,328659 & 40,784 \\
\hline Sevilla & 1,928962 & 0,703021 & 36,446 \\
\hline Puente Genil & 0,805857 & 0,030424 & 3,775 \\
\hline Antequera & 1,625827 & 0,041854 & 2,574 \\
\hline Málaga & 1,625827 & 0,568030 & 34,938 \\
\hline Guadalajara & 0,256461 & 0,084453 & 32,930 \\
\hline Calatayud & 0,973325 & 0,020837 & 2,141 \\
\hline Zaragoza & 0,973325 & 0,674725 & 69,322 \\
\hline Lleida & 0,442308 & 0,138416 & 31,294 \\
\hline Tarragona & 0,811401 & 0,134085 & 16,525 \\
\hline Barcelona & 5,529099 & 1,615448 & 29,217 \\
\hline Tardienta & 0,228361 & 0,000998 & 0,437 \\
\hline Huesca & 0,228361 & 0,052443 & 22,965 \\
\hline Segovia & 0,164169 & 0,055200 & 33,636 \\
\hline Valladolid & 0,534874 & 0,313437 & 58,600 \\
\hline Cuenca & 0,219138 & 0,056703 & 25,875 \\
\hline Albacete & 0,402318 & 0,171390 & 42,601 \\
\hline Requena-Utiel & 2,578719 & 0,021394 & 0,830 \\
\hline Valencia & 2,578719 & 0,798033 & 30,947 \\
\hline A Coruña & 1,147124 & 0,246028 & 21,447 \\
\hline Santiago de Compostela & 1,147124 & 0,095207 & 8,300 \\
\hline Ourense & 0,333257 & 0,108002 & 32,408 \\
\hline
\end{tabular}

Fuente: INE y elaboración propia.

Este cálculo sirve para determinar el grado de macrocefalia o, por el contrario, policentrismo, existente en el sistema de asentamientos urbanos de cada unidad provincial. 
Cabe destacar en este sentido el sistema macrocefálico de Aragón, con la hegemonía de Zaragoza respecto al resto de núcleos, hegemonía que lógicamente se acentúa si únicamente consideramos la masa demográfica de la provincia homónima, constituyendo casi un $70 \%$ de la población provincial.

En el caso opuesto se sitúa la provincia de Ciudad Real. Aunque la masa demográfica existente es menor, hay que considerar especialmente la no existencia de núcleos preponderantes en cuanto a masa poblacional. Ciudad Real capital únicamente alberga a poco más del $14 \%$ de la población provincial. El otro núcleo provincial con estación es Puertollano, con una masa menor pero muy similar, constituyendo cerca del $10 \%$ de la población total de la provincia.

Esta distribución puede darnos unas primeras pistas sobre potenciales flujos de usuarios de alta velocidad, teniendo en cuenta a su vez que una mayor masa demográfica posibilita una mayor variedad de servicios y de infraestructuras de transporte, por tanto, una mayor capacidad de atracción y recepción de usuarios, lo que se traduce en un mayor dinamismo. Estas diferencias pueden influir en la creación de unos patrones diferenciados de comportamiento de los usuarios en relación a sus preferencias a la hora de desplazarse.

Amén de los datos demográficos de los núcleos de población dotados de estación, un primer acercamiento más concreto a la hora de analizar la red española consiste en estudiar la relación de enlaces reales y directos entre estaciones, es decir, a cuántos nodos nos podemos dirigir desde una determinada estación y con, al menos, un tren diario por sentido.

La información se presenta en forma de matriz, señalando con el símbolo • aquellas conexiones existentes en la actualidad.

El orden de los nodos no es aleatorio, sino que está organizado en corredores, de tal forma que el primero de ellos es Madrid, seguido por Toledo y estaciones de la LAV hacia Sevilla. Los siguientes tres nodos hacen referencia al corredor Córdoba-Málaga (Puente Genil, Antequera y la propia Málaga). En tercer lugar, la LAV MadridBarcelona, seguida por la línea Zaragoza-Huesca, Madrid-Valladolid, corredor del Levante (hacia Valencia) y, finalmente, la LAV gallega, con las estaciones de A Coruña, Santiago de Compostela y Ourense.

Con esta estructura podemos identificar relaciones de enlace en torno a las agrupaciones de símbolos $\bullet$. Observando el cuadro 2 pueden extraerse los siguientes rasgos identificatorios:

1. Madrid, por su capitalidad y su situación central, se configura como el nodo con mayor número de conexiones. La radialidad del sistema permite viajar desde esta ciudad al resto de nodos de la red, a excepción de la LAV gallega, ya que

ISSN: 0212-8594 ISSN-e: 2340-2776. № DOI: http://dx.doi.org/10.12795/rea.2013.i30.04

REA 30 (2013): 78-101

http://www.publius.us.es/estudios_andaluces 
hasta la fecha de redacción del presente trabajo, todavía no existe conexión en corredor de alta velocidad entre la capital y Galicia.

2. Pueden identificarse gracias a la concentración de símbolos, los dos corredores principales (el sur y el noreste). De hecho, también se observa como ambas líneas están interconectadas. Por ejemplo, existe enlace directo entre Sevilla y Barcelona, sin pasar por Madrid capital. Sin embargo, el vacío existente entre ambas agrupaciones de símbolos hace referencia a que las estaciones de Calatayud y Guadalajara, ambas en el corredor noreste, no presentan esta característica, es decir, desde ambas es posible dirigirse hacia Madrid y también hacia Barcelona y resto de estaciones intermedias, pero no hacia Córdoba o Málaga, por ejemplo.

\section{Cuadro 2. Matriz de enlaces reales entre nodos}

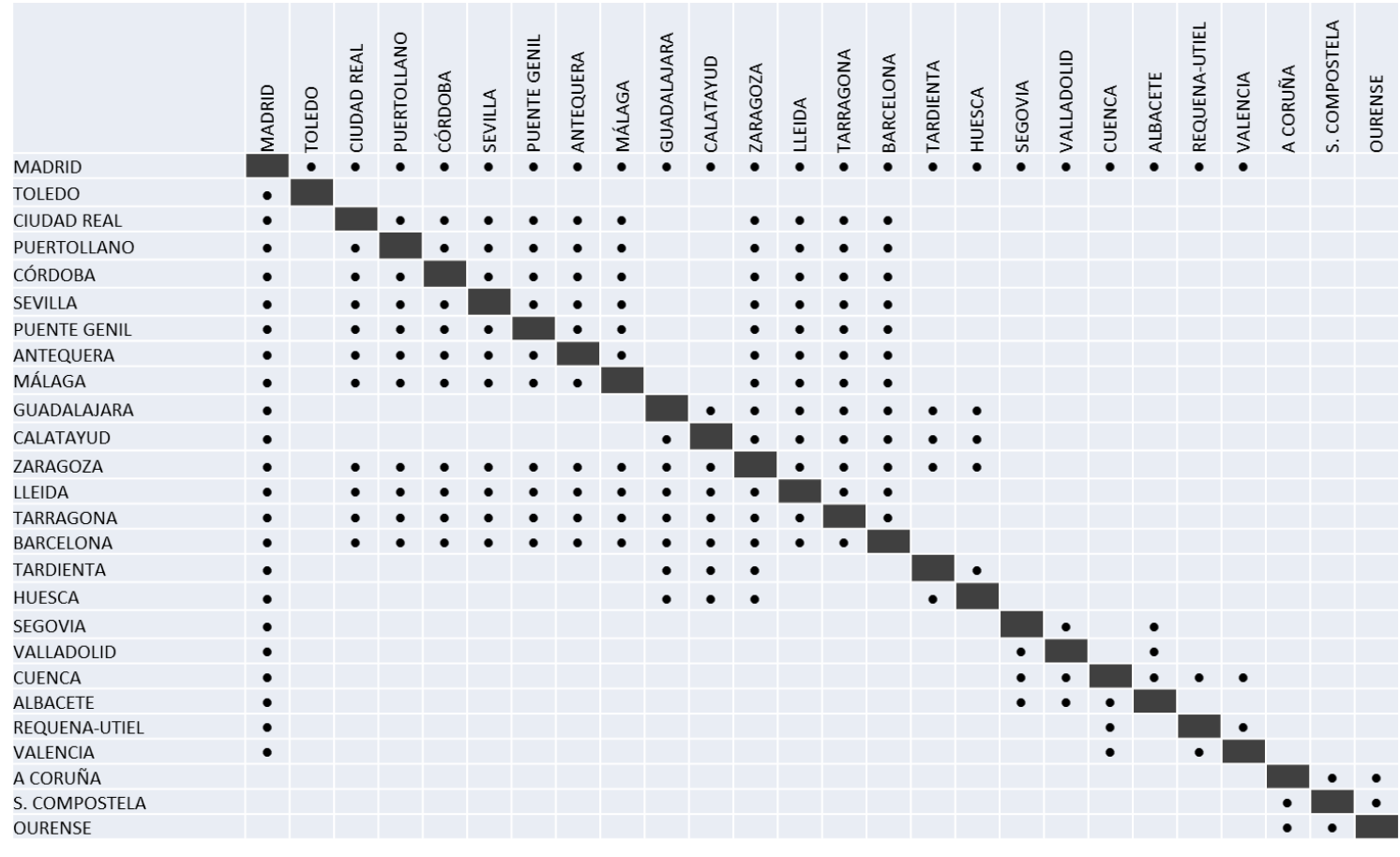

Fuente: Elaboración propia.

3. En el resto de corredores la densidad del punteado es mucho menor. Esto significa que existen menos enlaces, limitándose en muchos de los casos a los nodos más próximos a la línea diagonal divisoria de la matriz, es decir, a los enlaces consecutivos.

4. La matriz es cuasi simétrica. En todos los casos puede realizarse el desplazamiento entre dos nodos conectados en ambos sentidos, excepto en las conexiones entre la LAV Madrid-Valladolid con Cuenca. No existen posibilidades de desplazamiento en alta velocidad desde Segovia ni desde

ISSN: 0212-8594 ISSN-e: 2340-2776. № DOI: http://dx.doi.org/10.12795/rea.2013.i30.04 
Valladolid con la ciudad manchega, aunque sí desde esta última a ambas estaciones, mediante servicios Alvia.

Una vez discriminados los enlaces indirectos, la siguiente fase ha consistido en la creación de una segunda matriz en la cual aparecen los datos relativos a las distancias kilométricas existentes entre todos los enlaces computados (Véase cuadro 3).

A tal efecto se han utilizado datos aportados por el Administrador de Infraestructuras Ferroviarias (ADIF), entre otras fuentes; aunque para determinados enlaces se ha recurrido a cálculos propios a partir de datos preexistentes. Por ejemplo, la distancia entre Madrid y Sevilla es de $471 \mathrm{Km}$., por tanto, si se sabe qué distancia existe entre Córdoba y Sevilla también puede averiguarse la correspondiente al trayecto MadridCórdoba mediante una simple resta.

Cuadro 3. Matriz de distancias entre nodos para conexiones de alta velocidad (Km.).

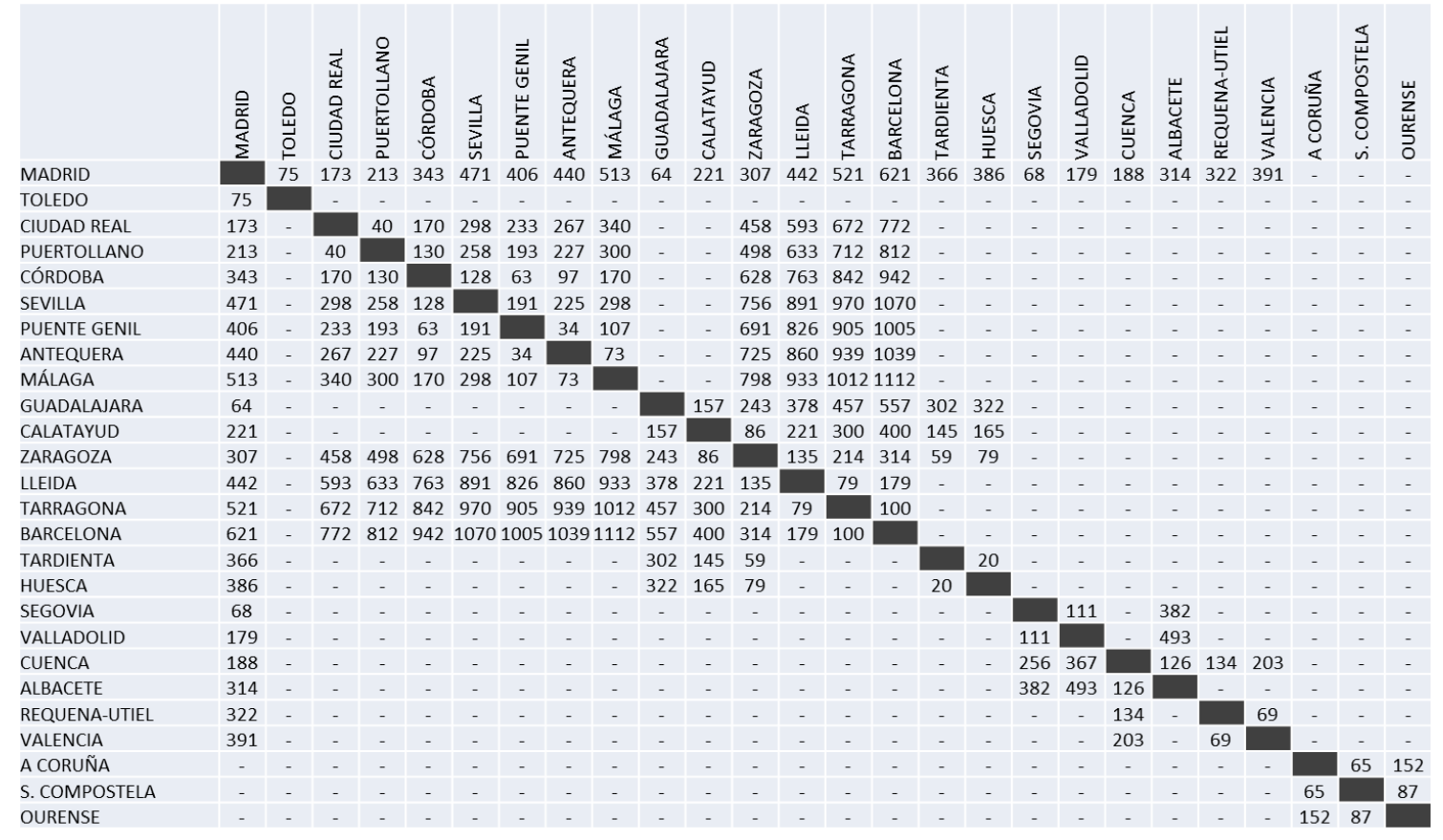

Fuente: RENFE, prensa y elaboración propia.

Sin embargo, no en todos los casos la operación es tan sencilla. Nos referimos a los trayectos entre el corredor sur y el corredor noreste. Por ejemplo para un trayecto Sevilla-Barcelona no es simplemente sumar la distancia entre Sevilla-Madrid a la de Madrid-Barcelona puesto que los trazados no son exactamente esos. De hecho, viniendo desde el sur, a partir de Ciudad Real la siguiente parada no es en Madrid sino en Zaragoza; y no sólo es que no pare en Madrid, sino que ni siquiera pasa por la capital. Los trenes que realizan estos recorridos, antes de llegar a la estación de Madrid-Puerta de Atocha se desvían hacia el este, en el denominado by-pass de

ISSN: 0212-8594 ISSN-e: 2340-2776. № DOI: http://dx.doi.org/10.12795/rea.2013.i30.04

REA 30 (2013): 78-101

http://www.publius.us.es/estudios_andaluces 
Perales del Río (Getafe), a partir del cual ya enlazan con la LAV Madrid-Barcelona. Esto implica un cierto ahorro de distancia, de algo más de $20 \mathrm{~km}$. en total, reduciéndose a su vez el tiempo de viaje.

\section{EL MODELO GRAVITATORIO Y LA APLICACIÓN A LA RED.}

Para establecer unas primeras diferencias, se decidió estructurar los enlaces objeto de estudio en tres niveles o categorías, teniendo en cuenta el número de estaciones existentes en cada una de las provincias y el tipo de enlace resultante de su combinación.

A la hora de la aplicación de las distancias, se han tenido en cuenta las referidas en el cuadro 4, sin embargo, en cuanto a masas demográficas, el criterio ha variado dependiendo de la casuística anterior.

Cuadro 4. Tipología de enlaces de la red de alta velocidad.

\begin{tabular}{|l|l|l|}
\hline CASO & EJEMPLO & DESCRIPCIÓN \\
\hline 1 & Tarragona-Barcelona & Dos provincias con una sola estación \\
\hline 2 & Ciudad Real-Puertollano & $\begin{array}{l}\text { Ambas ciudades pertenecen a la misma provincia } \\
\text { y cuentan con estación }\end{array}$ \\
\hline 3 & Guadalajara-Calatayud & $\begin{array}{l}\text { Una provincia con una estación (Guadalajara) y } \\
\text { otra con dos estaciones (Zaragoza) }\end{array}$ \\
\hline
\end{tabular}

Fuente: Elaboración propia.

Concretamente, para el caso 1 , se han aplicado los valores demográficos totales de la provincia, puesto que ambas cuentan con una única estación ${ }^{3}$. Es el caso más sencillo a la hora de aplicar posteriormente el modelo, puesto que se entiende que la mayor parte de los usuarios potenciales del tren residentes en una de estas provincias van a subirse a éste en la estación de su provincia. No obstante, y como es de suponer, el criterio político-administrativo no rige inexorablemente estas pautas. Siempre existirán casos en los que un usuario residente en un municipio cercano al límite fronterizo con otra provincia, se suba al tren en la estación más cercana, aunque ésta esté emplazada en la provincia contigua. Por ejemplo, a un usuario residente en Meco (Madrid), le resulta más cercana la estación de Guadalajara-Yebes que Madrid-Puerta de Atocha.

Para el caso 2 hay que considerar que ambas estaciones se encuentran relativamente próximas. Por consiguiente, lo que se producirá es un reparto de la demanda provincial de usuarios, aunque, como ya se ha comentado para el caso anterior, la demanda no tiene porqué ajustarse a los límites político-administrativos.

\footnotetext{
${ }^{3}$ En el caso de Madrid, funciona como un único nodo excepto para las conexiones entre la LAV MadridValladolid con Cuenca y Albacete, siendo éste el único corredor en el que los trenes realizan doble parada en Madrid. Por tanto, desde un punto de vista demográfico, para la capital se aplica la cifra provincial.
}

ISSN: 0212-8594 ISSN-e: 2340-2776. № DOI: http://dx.doi.org/10.12795/rea.2013.i30.04

REA 30 (2013): 78-101

http://www.publius.us.es/estudios_andaluces 
Partiendo de la hipótesis de que sí se ajuste a dichos límites, es decir, que la demanda potencial que capten las estaciones de los dos núcleos en cuestión sea la totalidad de la masa demográfica de la provincia, nos surge una segunda dificultad: Cómo delimitar el área de influencia de cada estación dentro de la provincia.

Para ello hay que considerar la enorme heterogeneidad existente en España en cuanto a las masas demográficas de las capitales respecto a la población total de sus respectivas provincias (Véase cuadro 1 ).

Con todo esto, ¿qué área de influencia debe considerarse para cada una de las estaciones de los tramos de tipo 2 ? Debido a las dificultades detectadas a la hora de establecer un búfer, se ha decidido otorgarle la mayor parte del peso provincial a la capital de la misma, captando todo el tráfico de viajeros provinciales excepto los de los residentes en el otro núcleo con estación.

\section{Cuadro 5. Matriz de coeficientes gravitatorios.}

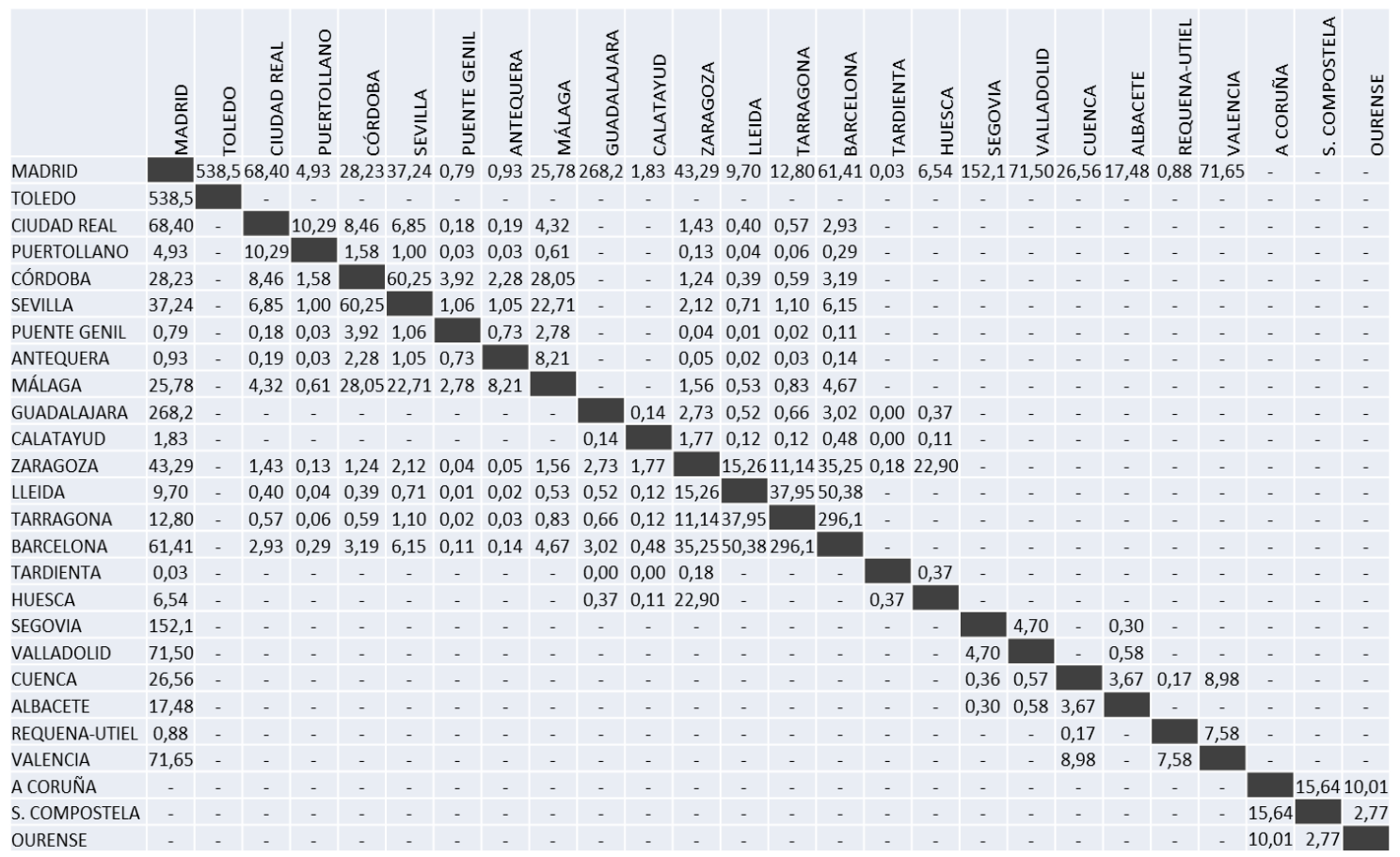

Fuente: Elaboración propia.

Esto implica que los resultados en provincias como la de Ciudad Real pueden quedar algo distorsionados. No obstante, también debe afirmarse que la capital provincial tiene un punto a su favor respecto a Puertollano como es su mayor cercanía a Madrid. Aunque Puertollano se sitúa más cerca de Córdoba y Sevilla, debe considerarse a otro nivel el poder de atracción de flujos de la capital nacional. 
Por último, el caso 3 es una mezcla de los otros dos. Un ejemplo explicativo es el de Guadalajara-Calatayud. A la hora de escoger las masas demográficas se elegirán la de la provincia de Guadalajara, al ser esta capital el único núcleo provincial que cuenta con alta velocidad; y la del término municipal de Calatayud.

Llegados a este punto, una vez recopilados los datos anteriores relativos a masas demográficas y distancias kilométricas para los enlaces directos, ya es posible la aplicación de la fórmula expuesta en el apartado 2, para la obtención de los coeficientes gravitatorios. Los resultados se incluyen en el cuadro 5.

Como puede observarse, se ha calculado el coeficiente gravitacional para cada enlace o tramo, sea consecutivo o no. De esta forma, todos los valores que nos dan resultan de la utilización de un único tren, para así poder integrar y representar de forma más unitaria los valores resultantes. También se han tenido en cuenta la tipología de casos anteriormente expuesta a la hora de tomar unos $u$ otros datos demográficos de referencia.

Cuadro 6. Categorización de los valores del Coeficiente Gravitatorio para los tramos de alta velocidad

\begin{tabular}{|c|c|c|}
\hline TIPO & MASA & PROXIMIDAD \\
\hline 1 & Alta & Baja \\
\hline 2 & Alta & Alta \\
\hline 3 & Baja \\
\hline 4 & \multicolumn{2}{|c|}{ Resto de valores } \\
\hline
\end{tabular}

Fuente: Elaboración propia.

Analizando ya detalladamente los valores resultantes, lo que primeramente llama la atención es que son tremendamente dispares, desde los 538,5 del tramo MadridToledo hasta una importante multiplicidad de valores menores a la unidad.

Esta disparidad es fruto del gran desequilibrio existente entre masas demográficas de cada pareja de nodos considerada así como del papel de la distancia espacial que los separa. Teniendo en cuenta ambos criterios, se ha establecido una categorización de los valores resultantes, siguiendo el siguiente esquema:

Siendo:

- Masa demográfica alta: Al menos uno de los nodos cuenta con un volumen de habitantes superior al millón. Por tanto, es masa baja si no se alcanza dicha cifra sumando las masas de ambos nodos.

- Proximidad: Se entiende como proximidad alta aquella distancia espacial existente entre dos nodos considerados menor a $200 \mathrm{~km}$, y viceversa. 
Para aquellos enlaces cuyo CG es menor a 10 , se ha obviado su inclusión en esta categorización, debido a su escaso valor de potencial gravitatorio, agrupándolos en un último cuarto grupo.

En las tres tablas siguientes (cuadros 7,8 y 9) se exponen los resultados de la aplicación del modelo. En cada uno de ellos se muestran los enlaces correspondientes a cada tipo establecido en el anterior cuadro 6, ordenados de mayor a menor coeficiente de gravedad, indicando además la masa demográfica de cada par de nodos y la distancia kilométrica comprendida entre ambos.

Está compuesto de aquellos desplazamientos que podrían considerarse como más rentables y/o competitivos dentro de la actual red de alta velocidad. A saber:

Madrid-Valencia.

El corredor hacia Valencia es uno de los de más reciente creación y se prevé que el componente turístico sea uno de los motivos fundamentales de viaje. Posee el valor más alto del grupo, ayudado por una importante masa demográfica ( 9 millones de

habitantes entre ambas provincias) y una distancia moderada, por debajo de los 400 $\mathrm{km}$.

Madrid - Barcelona

Es claramente uno de los corredores más factibles a la hora de aprovechar las ventajas de la alta velocidad y disponer de un transporte competitivo frente al automóvil y el avión. Cuenta además con el segundo valor más alto dentro del tipo 1.

Podrá ser un eje fundamental en las comunicaciones ferroviarias de las dos ciudades más pobladas de España con la futura conexión a Francia. Está considerado además de interés prioritario para la UE. Se trata de dos ciudades ( $y$, por extensión, dos provincias) con masa demográfica elevada y separadas por una distancia de unos 600 $\mathrm{Km}$.

Así, confluyen dos factores claves: demanda potencial muy relevante (más de 11 millones de habitantes entre ambas provincias y casi 5 si hablamos sólo de los términos municipales) y distancia espacial entre 300 y 700 Km., suficientemente más largo para acortar tiempos respecto al vehículo privado y no demasiado distantes como para que el avión obtenga claras ventajas. 
Cuadro 7. Tabla síntesis de coeficientes gravitacionales. Tipo 1.

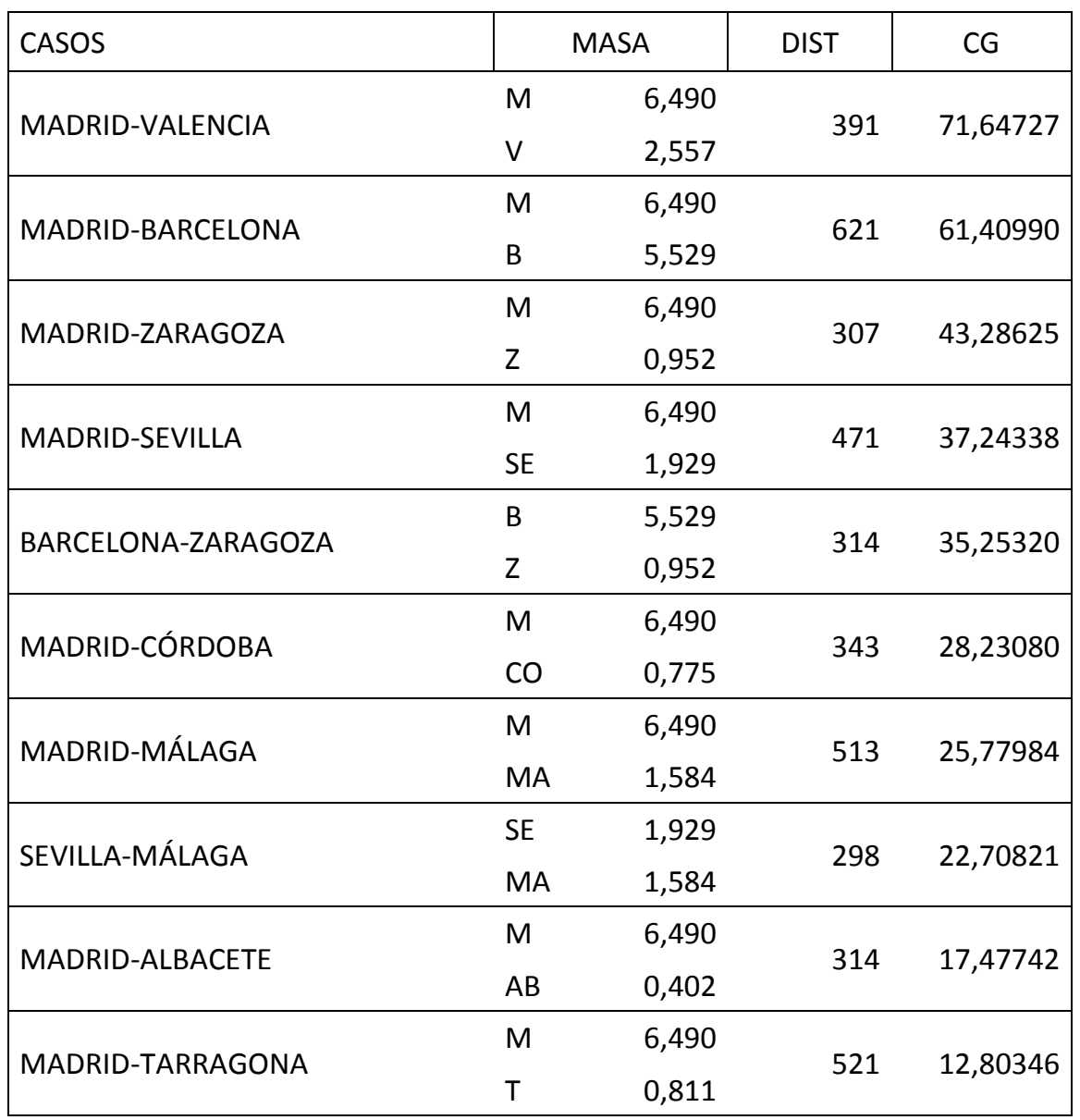

Fuente: Elaboración propia.

Madrid-Zaragoza

Este tramo del corredor Madrid - Barcelona se sitúa en el umbral mínimo a partir del cual se considera, según el PEIT, que el AVE puede ser rentable y competitivo, ya que ambas ciudades están situadas a 300 Kilómetros. Obtiene un valor bastante alto, el tercero más elevado de este tipo. La gran masa demográfica madrileña y la distancia moderada explican este hecho.

De igual forma, conviene destacar la estructura del poblamiento aragonés y, más en concreto, zaragozano; con la importante macrocefalia existente: la ciudad de Zaragoza alberga al $70 \%$ de la población provincial, siendo un importante nodo industrial y de servicios, turístico, sede de la Academia Militar, etc.

\section{Madrid-Sevilla}

El primer enlace de alta velocidad español posee un importante componente turístico y de negocios. Sevilla posee mayor masa demográfica que Zaragoza, pero en este caso

ISSN: 0212-8594 ISSN-e: 2340-2776. № DOI: http://dx.doi.org/10.12795/rea.2013.i30.04 
Cuadro 8. Tabla síntesis de coeficientes gravitacionales. Tipo 2.

\begin{tabular}{|c|c|c|c|c|}
\hline CASOS & & & DIST & CG \\
\hline \multirow{2}{*}{ MADRID-TOLEDO } & M & 6,490 & \multirow{2}{*}{75} & \multirow{2}{*}{538,53351} \\
\hline & TO & 0,707 & & \\
\hline \multirow{2}{*}{ BARCELONA-TARRAGONA } & B & 5,529 & \multirow{2}{*}{100} & \multirow{2}{*}{296,09689} \\
\hline & $\mathrm{T}$ & 0,811 & & \\
\hline \multirow{2}{*}{ MADRID-GUADALAJARA } & M & 6,490 & \multirow{2}{*}{64} & \multirow{2}{*}{268,18137} \\
\hline & GU & 0,256 & & \\
\hline \multirow{2}{*}{ MADRID-SEGOVIA } & M & 6,490 & \multirow{2}{*}{68} & \multirow{2}{*}{152,06895} \\
\hline & SG & 0,164 & & \\
\hline \multirow{2}{*}{ MADRID-VALLADOLID } & M & 6,490 & \multirow{2}{*}{179} & \multirow{2}{*}{71,50109} \\
\hline & VA & 0,535 & & \\
\hline \multirow{2}{*}{ MADRID-CIUDAD REAL } & M & 6,272 & \multirow{2}{*}{173} & \multirow{2}{*}{68,40379} \\
\hline & CR & 0,478 & & \\
\hline \multirow{2}{*}{ CÓRDOBA-SEVILLA } & $\mathrm{CO}$ & 0,775 & \multirow{2}{*}{128} & \multirow{2}{*}{60,25484} \\
\hline & SE & 1,929 & & \\
\hline \multirow{2}{*}{ BARCELONA-LLEIDA } & B & 5,529 & \multirow{2}{*}{179} & \multirow{2}{*}{50,37523} \\
\hline & $\mathrm{L}$ & 0,442 & & \\
\hline \multirow{2}{*}{ LLEIDA-TARRAGONA } & $\mathrm{L}$ & 0,442 & \multirow{2}{*}{79} & \multirow{2}{*}{37,95335} \\
\hline & $\mathrm{T}$ & 0,811 & & \\
\hline \multirow{2}{*}{ CÓRDOBA-MÁLAGA } & $\mathrm{CO}$ & 0,775 & \multirow{2}{*}{170} & \multirow{2}{*}{28,05034} \\
\hline & MA & 1,584 & & \\
\hline \multirow{2}{*}{ MADRID-CUENCA } & M & 6,490 & 100 & 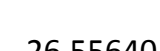 \\
\hline & $\mathrm{CU}$ & 0,219 & 188 & 20,55040 \\
\hline & Z & 0,952 & & \\
\hline ZARAGULA-HUESCA & $\mathrm{H}$ & 0,227 & 19 & $\angle 2,901 / 1$ \\
\hline$\hat{A}$ & C & 1,052 & & \\
\hline ACUNUINAT-S. CUIVITUSILLA & SC & 0,095 & כ & $10,044 / 1$ \\
\hline 7АRAGO7A-IIFIDA & Z & 0,952 & 135 & 1525670 \\
\hline СANAUULA-LLEIUA & $\mathrm{L}$ & 0,442 & 100 & $10,250 / 0$ \\
\hline RIÑA_RI & C & 1,052 & $5 ?$ & 123 \\
\hline 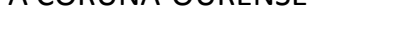 & OU & 0,333 & 年 & 1) \\
\hline
\end{tabular}

Fuente: Elaboración propia.

la distancia mayor $(471 \mathrm{Km}$. frente a 307$)$ penaliza el valor final del coeficiente de gravedad. 
Cuadro 9. Tabla síntesis de coeficientes gravitacionales. Tipo 3.

\begin{tabular}{|c|c|c|c|c|}
\hline CASOS & & & DIST & CG \\
\hline \multirow{2}{*}{ C. REAL-PUERTOLLANO } & $\mathrm{CR}$ & 0,478 & \multirow{2}{*}{40} & \multirow{2}{*}{10,29200} \\
\hline & PL & 0,052 & & \\
\hline \multirow{2}{*}{ CUENCA-ALBACETE } & $\mathrm{CU}$ & 0,219 & \multirow{2}{*}{126} & \multirow{2}{*}{3,66514} \\
\hline & $A B$ & 0,402 & & \\
\hline \multirow{2}{*}{ S. COMPOSTELA-OURENSE } & SC & 0,095 & \multirow{2}{*}{87} & \multirow{2}{*}{2,76665} \\
\hline & OU & 0,333 & & \\
\hline \multirow{2}{*}{ P.GENIL-ANTEQUERA } & PG & 0,030 & \multirow{2}{*}{34} & \multirow{2}{*}{0,72701} \\
\hline & $A Q$ & 0,042 & & \\
\hline \multirow{2}{*}{ TARDIENTA-HUESCA } & TT & 0,001 & \multirow{2}{*}{20} & \multirow{2}{*}{0,37440} \\
\hline & $\mathrm{H}$ & 0,227 & & \\
\hline \multirow{2}{*}{ ZARAGOZA-TARDIENTA } & Z & 0,952 & \multirow{2}{*}{59} & \multirow{2}{*}{0,18023} \\
\hline & TT & 0,001 & & \\
\hline \multirow{2}{*}{ CUENCA-REQUENA-UTIEL } & CU & 0,219 & \multirow{2}{*}{134} & \multirow{2}{*}{0,17232} \\
\hline & $\mathrm{RQ}$ & 0,021 & & \\
\hline \multirow{2}{*}{ GUADALAJARA-CALATAYUD } & GU & 0,134 & \multirow{2}{*}{157} & \multirow{2}{*}{0,14309} \\
\hline & $\mathrm{CY}$ & 0,057 & & \\
\hline
\end{tabular}

Fuente: Elaboración propia.

\section{Barcelona-Zaragoza}

El carácter del enlace es similar al Madrid-Zaragoza. La distancia es prácticamente idéntica (314 Km. frente a 307), por tanto el hecho de que el valor final sea algo menor se debe a la masa demográfica de Barcelona, muy importante pero cerca de un millón de habitantes inferior a la madrileña.

\section{Madrid-Córdoba, Madrid-Málaga y Sevilla-Málaga}

Los enlaces de Madrid con el resto de capitales andaluzas dan un valor gravitatorio similar, así como la conexión Sevilla-Málaga. La menor distancia existente entre Madrid y Córdoba respecto a Málaga queda compensada en buena medida por la mayor masa de esta última. Debe destacarse la importancia de Córdoba como intersección entre el corredor suroccidental a Sevilla y el meridional a Málaga; y de ésta última como centro de servicios de nuevas tecnologías y foco atractor de turismo.

\section{Madrid-Albacete y Madrid-Tarragona}

Estos enlaces son los que poseen unos valores más bajos. Respecto a Albacete, el valor se penaliza sobre todo por la baja masa demográfica de la provincia manchega, pese a que Albacete sea la ciudad más poblada de Castilla-La Mancha. Por su parte, Tarragona posee un sistema de poblamiento más policéntrico (es una de las capitales con menor

ISSN: 0212-8594 ISSN-e: 2340-2776. № DOI: http://dx.doi.org/10.12795/rea.2013.i30.04 
cuantía de población respecto a su provincia), con casi el doble de masa provincial que Albacete; sin embargo en este caso el factor penalizador del valor es la distancia, superior a los $500 \mathrm{~km}$.

En este segundo bloque se han insertado aquellos tramos que parten, en su mayor parte, desde Madrid o Barcelona, igual que en el anterior. Sin embargo, existe una diferencia fundamental: en este caso la proximidad juega un papel crucial a la hora de explicar los valores de CG resultantes.

En todos los casos, al menos uno de los nodos tiene una población potencial inferior al millón de habitantes. Se han agrupado aquellos tramos que conectan una gran ciudad con la que se sitúa consecutiva a ésta. Los tramos que parten desde Madrid o Barcelona son así los más representativos.

Nos encontramos con un tipo de enlaces cuyos valores de CG son muy dispares. Esto es debido tanto a los distintos valores de masa demográfica como, sobre todo, a la escasa distancia existente entre ambos nodos en todos los casos: siempre es inferior a $200 \mathrm{Km}$. Al ser tan baja, el valor de denominador es igualmente bajo, con lo cual los valores resultantes de CG son abultados en muchos de ellos.

\section{Madrid-Toledo}

El valor más alto de todo el análisis corresponde a este tramo: 538,5. Se debe a la alta masa demográfica de Madrid junto con una población de Toledo (provincia) relativamente elevada. Sin embargo, el factor clave y más explicativo de dicha cifra es la escasa distancia existente $(75 \mathrm{Km})$.

\section{Barcelona-Tarragona}

Corresponde al segundo valor absoluto más alto, destacando la masa demográfica barcelonesa, con 5,5 millones de habitantes. Pese a ser superior la masa de Tarragona respecto a la de Toledo, la distancia espacial es algo mayor, por lo que el valor del CG, aun siendo alto (296), resulta poco más de la mitad del valor del corredor anterior.

\section{Madrid-Guadalajara, Madrid-Segovia}

Ambos enlaces constituyen nodos consecutivos de Madrid y están situados a menos de $100 \mathrm{Km}$. De hecho la distancia es sensiblemente menor a la de Barcelona-Tarragona, especialmente para Madrid-Guadalajara, aunque la menor masa demográfica penaliza el valor final, especialmente para la conexión con Segovia puesto que esta provincia posee una de las masas de población menores de toda España.

ISSN: 0212-8594 ISSN-e: 2340-2776. № DOI: http://dx.doi.org/10.12795/rea.2013.i30.04

REA 30 (2013): 78-101

http://www.publius.us.es/estudios_andaluces 


\section{Madrid-Valladolid, Madrid-Ciudad Real}

Poseen valores de CG muy parejos, puesto que las masas son similares y las distancias prácticamente idénticas, si bien es cierto que al existir en Valladolid una única estación de AV todo el potencial tráfico de viajeros se dirigirá hacia la estación de Campo Grande, mientras que en la provincia de Ciudad Real, al existir dos estaciones, parte del tráfico será absorbido por Puertollano.

\section{Córdoba-Sevilla, Córdoba-Málaga}

El enlace Córdoba-Sevilla obtiene mayor valor debido a la masa demográfica de Sevilla, superior a la malacitana. También juega a su favor la menor distancia entre los nodos (128 Km. frente a 170).

\section{Zaragoza-Huesca}

Pese a estar muy próximas, el valor es ciertamente bajo debido sobre todo a la escasa masa demográfica oscense, una de las menores de todo el territorio nacional, con poco más de 220.000 habitantes.

\section{A Coruña-Santiago de Compostela, A Coruña-Ourense}

Todos los valores del corredor gallego resultan bajos, aunque el motivo es diferente según el caso. Para el tramo A Coruña-Santiago el valor es algo superior al de la conexión entre A Coruña y Ourense debido a que los primeros son nodos consecutivos, separados sólo por $65 \mathrm{Km}$., mientras que para el segundo caso la distancia rebasa los $150 \mathrm{Km}$. La consideración de la totalidad de la masa provincial para Ourense matiza el resultado aunque el valor del coeficiente sigue siendo menor.

Los tramos encuadrados en el tercer tipo se asemejan a los del segundo en el sentido en que existe una gran proximidad entre los nodos. Sin embargo, las masas demográficas son muy pequeñas (siempre menores a 100.000 habitantes), haciendo referencia a pequeñas capitales de provincia o núcleos no capitales.

Llegados a este punto, se deduce que las señas de identidad son: núcleos pequeños y muy próximos. Los valores resultantes son, por consiguiente, bajos en todos los casos, por debajo de 10 en todos los casos incluidos a excepción del tramo Ciudad RealPuertollano.

Llama la atención casos como los de Tardienta-Huesca, en relación a las distancias (escasos $20 \mathrm{Km}$.) o Puente Genil-Antequera, cuya suma de masas demográficas apenas supera los 70.000 habitantes. Los valores son especialmente llamativos para el municipio tardientano, dada su bajísima masa demográfica (en torno a 1.000

ISSN: 0212-8594 ISSN-e: 2340-2776. № DOI: http://dx.doi.org/10.12795/rea.2013.i30.04 
habitantes). Esta cifra anula la influencia que pueda ejercer la variable distancia sobre el coeficiente de gravedad, ya que tanto su conexión con Huesca como con Zaragoza registran unos valores menores a la unidad.

Para representar espacialmente este conjunto de datos se ha elaborado un mapa (figura 2) en el que se ha calculado el promedio de valores de CG para cada nodo, matizando los importantes contrastes existentes derivados de masas y distancias.

A la vista del mapa, es muy claro el carácter radial de la red, por el peso de Madrid frente al resto. Toledo, Guadalajara y, en menor, medida, Segovia, poseen también valores altos pero son consecuencia directa también de la influencia de Madrid. En un segundo término podemos citar a Barcelona, también con importante masa demográfica que extiende su influencia hacia Tarragona y Lleida.

En un nivel inferior encontramos, en primer lugar, a las ciudades andaluzas, que forman su propio sistema policéntrico, con valores similares para Córdoba, Sevilla y Málaga; en segundo lugar, Zaragoza, aunque su influencia hacia Calatayud y Huesca es apenas visible; y en tercer lugar a Valencia, que pese a tener importante masa demográfica, los valores son más bien bajos al tener una única conexión con nodos muy poblados (Madrid), ya que aún no existe conexión en alta velocidad con ciudades como Alicante o Murcia, con masas demográficas muy superiores a las de Cuenca o Requena.

Tras la categorización anteriormente expuesta, una últimafase realizada ha sido el paso de valores de coeficiente gravitatorio a valores de pasajeros, para cada uno de los enlaces analizados. Los resultados aparecen en el cuadro 10.

Figura 2. Resultados del Modelo de Archer para cada nodo.

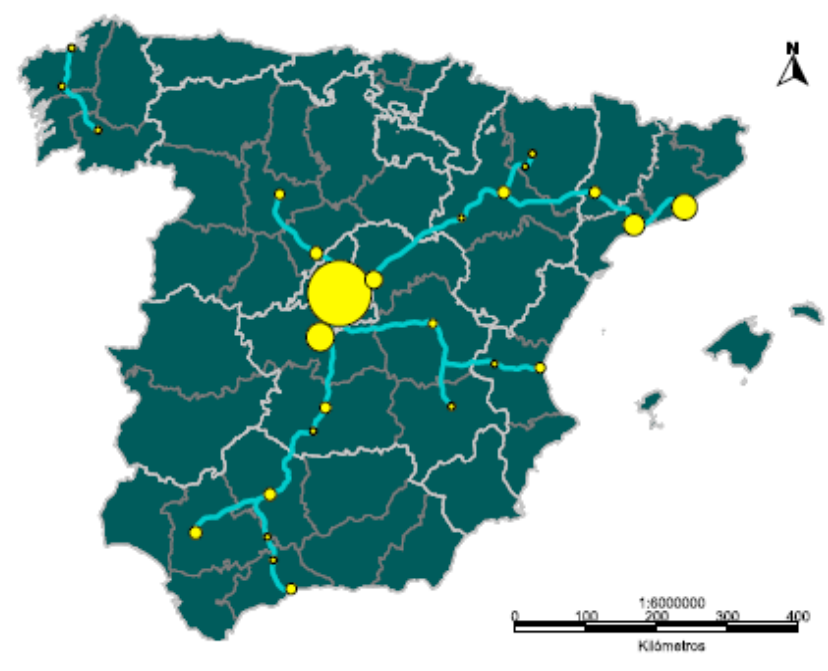

Fuente: Elaboración propia.

ISSN: 0212-8594 ISSN-e: 2340-2776. № DOI: http://dx.doi.org/10.12795/rea.2013.i30.04 REA 30 (2013): 78-101

http://www.publius.us.es/estudios_andaluces 


\section{Cuadro 10. Matriz de pasajeros máximos potenciales (millones anuales).}

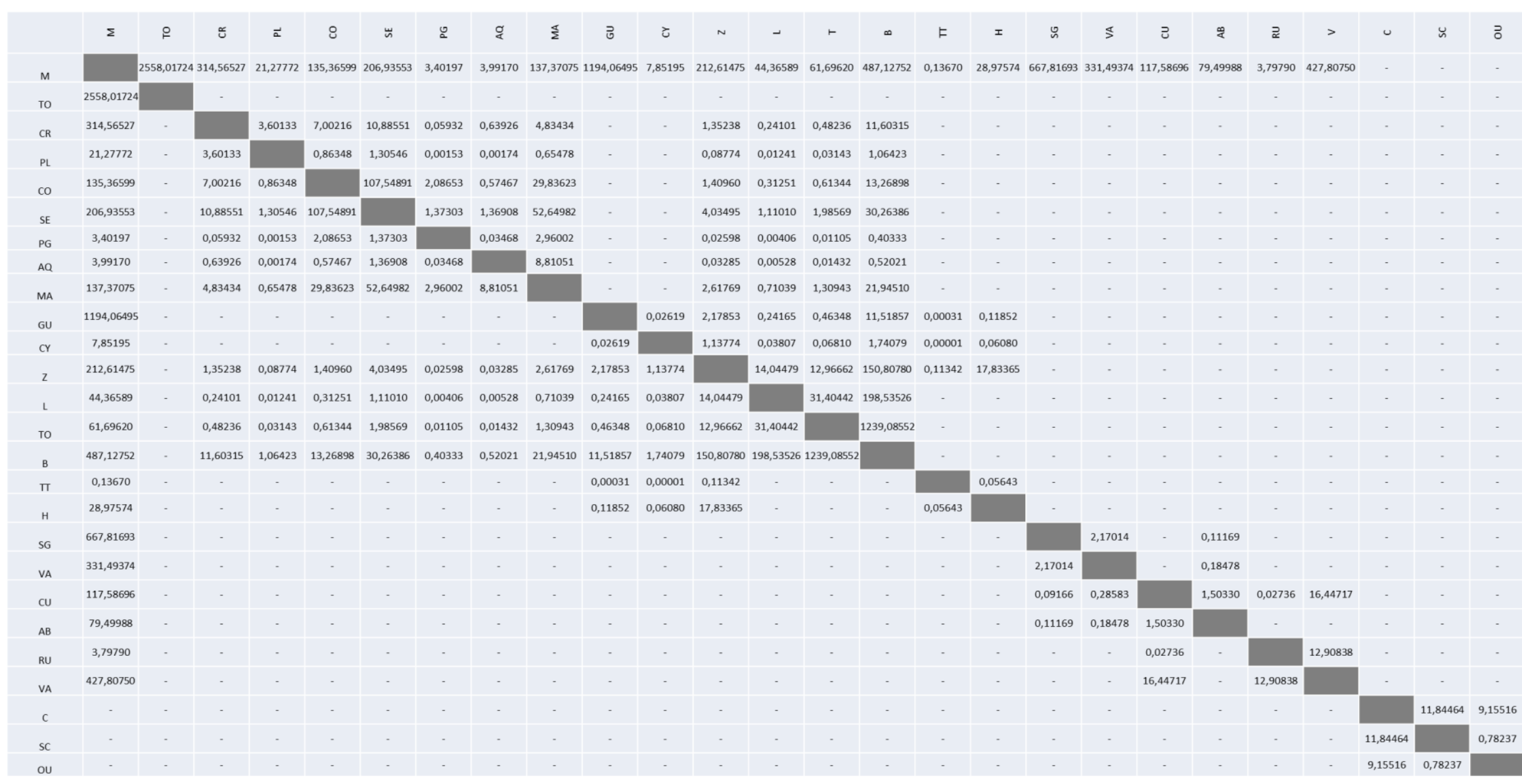

Fuente: Elaboración propia.

ISSN: 0212-8594 ISSN-e: 2340-2776. № DOI: http://dx.doi.org/10.12795/rea.2013.i30.04

REA 30 (2013): 78-101

http://www.publius.us.es/estudios_andaluces 
Para lograr una mayor precisión en los valores, se ha aumentado el número de decimales, lo cual ha necesitado el cambio de orientación de la matriz para lograr una mayor legibilidad de los datos.

Los valores resultantes son sensiblemente superiores a la de la matriz de coeficientes gravitatorios (cuadro 5) puesto que se están sumando las masas demográficas de ambas provincias o, según el caso, municipios, y se multiplica por el CG, que en muchos casos es mayor de 1.

Puede seguirse la misma tipificación de enlaces que en el paso anterior, aunque los valores resulten más contrastados. Concretamente, el valor más alto vuelve a ser Madrid-Toledo, con 2.558,017240 millones de usuarios. Lógicamente este valor tan abultado debe matizarse puesto que lo único que representa es la masa máxima anual potencial de viajeros que podrían desplazarse entre dichos nodos. En este sentido juega un papel esencial el peso de la masa demográfica madrileña dentro del corredor así como la escasa distancia espacial existente entre ambos nodos, que genera un coeficiente gravitatorio muy alto $y$, por ende, un volumen de viajeros potenciales también muy elevado.

Por otro lado, la mayor parte de los valores siguen siendo menores de 1 (millón de usuarios potenciales) debido a sus bajas masas demográficas, altas distancias o bien la confluencia de ambos factores, que generan un CG menor a la unidad y, por tanto, valores bajos.

No obstante, el nodo de Madrid prácticamente no cuenta con ningún valor menor a la unidad, lo que expresa su reforzamiento como nudo peninsular de comunicaciones ferroviarias en alta velocidad por su emplazamiento estratégico central. Masa demográfica muy potente y distancias nunca demasiado elevadas debido a esa posición central acentúan el carácter radial de la red y la preponderancia de la capital como foco generador y receptor de flujos de pasajeros.

\section{CONCLUSIONES.}

La red española de alta velocidad ferroviaria se encuentra en un proceso de continuo crecimiento, y las seis ciudades con mayor masa demográfica ya cuentan con este tipo de transporte. Si tenemos en cuenta las poblaciones provinciales, estamos hablando de más de 19 millones de habitantes. Es por ello que las implicaciones de su puesta en servicio y expansión son muy significativas y abordables desde múltiples perspectivas de análisis.

En el presente trabajo se ha pretendido arrojar unos primeros datos que determinen la cuantía de los flujos de usuarios en cada corredor de la red, teniendo en cuenta masas

ISSN: 0212-8594 ISSN-e: 2340-2776. № DOI: http://dx.doi.org/10.12795/rea.2013.i30.04

REA 30 (2013): 78-101

http://www.publius.us.es/estudios_andaluces 
demográficas y distancias entre nodos, mediante la aplicación y adaptación de un modelo gravitatorio derivado de estudios turísticos: el modelo de Archer.

Aunque sólo se han considerado masas y distancias, el modelo puede ganar precisión si se enriquece mediante la consideración de más variables, ya que hasta el momento es una aplicación de base amplia. De entre la multitud de variables potencialmente considerables podrían citarse: capacidad de renta, número de empresas existentes, presencia de plataformas logísticas cercanas, parques tecnológicos y empresariales, organización de eventos profesionales (congresos, jornadas, seminarios...), zonas de actividades de ocio, etc.; así como algunas atracciones particulares que se dan en determinados nodos. En definitiva, variables que incentiven y aumenten la capacidad de atracción de flujos de pasajeros.

No obstante, el rango tan amplio de valores resultantes reflejan, por un lado, el carácter radial de la red, favoreciendo a la capital por ser cabecera o fin de la mayor parte de las líneas $y$, por otro lado, los desequilibrios existentes en cuanto a masas demográficas se refiere dentro del territorio nacional.

De igual forma, también quedan reflejados otro hecho singular dentro de la concepción de la alta velocidad en España, como es la escasa distancia kilométrica existente entre algunas de las estaciones, muchas de ellas separadas por menos de cien kilómetros e, incluso, por menos de cincuenta. En este sentido, pese a existir escasa distancia entre ambas (favorable por tanto para un valor mayor del modelo), las escasas masas demográficas penalizan el dato final sobre potenciales usuarios, repercutiendo negativamente sobre la demanda y también sobre la oferta (frecuencia del servicio, viabilidad, etc.)

\section{BIBLIOGRAFÍA .}

Calderón, F.J. (2007): "Distrito turístico rural. Un modelo teórico desde la perspectiva de la oferta. Especial referencia al caso andaluz". Tesis Doctorales de Economía. Málaga, 585 pp.

Muñoz, A., Grande, F., Nuñez-Cacho, P. (1994): "Modelos de gravitación en el contexto turístico". IV Congreso de Economía Regional de Castilla y León. Burgos, Comunicaciones 3, pp. 1549-1562.

Pumain, D. (2004): "Modelo gravitatorio". Revista Hypergeo, sección "Análisis espacial".

www.renfe.es

ISSN: 0212-8594 ISSN-e: 2340-2776. № DOI: http://dx.doi.org/10.12795/rea.2013.i30.04

REA 30 (2013): 78-101

http://www.publius.us.es/estudios_andaluces 


\section{Sergio Martín Cabo}

La red española de alta velocidad ferroviaria: Análisis mediante un modelo gravitacional.

www.adif.es

www.ine.es

www.fomento.es/peit

ISSN: 0212-8594 ISSN-e: 2340-2776. № DOI: http://dx.doi.org/10.12795/rea.2013.i30.04 REA 30 (2013): 78-101

http://www.publius.us.es/estudios_andaluces 